\title{
CONHECIMENTOS GEOMÉTRICOS E ALGÉBRICOS DO TETRAEDRO FRACTAL 3D
}

\section{GEOMETRIC AND ALGEBRAIC KNOWLEDGE OF 3D FRACTAL TETRAHEDRON}

José Augusto da Costa Jacomeli, Fernando Pereira de Souza

Universidade Federal de Mato Grosso Do Sul - UFMS.

E-mail: gu jacomeli@outlook.com

RESUMO - Um fractal é uma figura que apresenta uma característica única que estará presente em todo o domínio da figura. Existem diversos tipos diferentes de fractais, alguns deles são construídos a partir de uma figura simples como um triângulo da geometria plana ou um tetraedro da geometria espacial. A partir da construção inicial de um fractal bidimensional começando com um triângulo equilátero e utilizando o Teorema de Napoleão, neste artigo, apresentamos uma construção de um novo fractal tridimensional utilizando ideias semelhantes ao Teorema de Napoleão em um tetraedro. Utilizando conceitos de geometria plana e espacial, esse fractal pode ser construído a partir de um tetraedro regular, e a partir dos pontos médios de suas arestas é construído um novo tetraedro com lado de razão $\frac{1}{2}$ em relação ao tetraedro inicial. Após essa construção, são estudadas as características do fractal aplicação infinita, como soma das áreas das superfícies e o volume total da figura formada.

Palavras-chave: Geometria; Fractal; Construção.

ABSTRACT - A fractal is a figure that has a unique characteristic that will be present in the entire domain of the figure. There are several different types of fractals, some of which are constructed from a simple figure such as a triangle of plane geometry or a tetrahedron of spatial geometry. From the initial construction of a two-dimensional fractal starting with an equilateral triangle and using Napoleon's Theorem, in this article, we present a construction of a new three-dimensional fractal using ideas similar to Napoleon's Theorem in a tetrahedron. Using concepts of plane and spatial geometry, this fractal can be built from a regular tetrahedron, and from the midpoints of its edges a new tetrahedron with a $1 / 2$ ratio side is built in relation to the initial tetrahedron. After this construction, the characteristics of the infinite application fractal are studied, such as the sum of the surface areas and the total volume of the formed figure.

Keywords: Geometry; Fractal; Construction. 


\section{INTRODUÇÃO}

Algumas das imagens fractais se assemelham a formas naturais tão de perto que a geometria fractal é considerada a geometria de objetos naturais. A visão predominante sobre o assunto, conforme expressa por Jurgens, Peitgen e Saupe (1990), é que:

Os Fractais são muito mais do que uma curiosidade matemática. Eles oferecem um método extremamente compacto para descrever objetos e formações. A geometria fractal parece descrever formas e formas naturais de forma mais graciosa e sucinta do que a geometria euclidiana.

Uma propriedade importante dos fractais é a auto-similaridade. Ao olhar atentamente para uma folha de samambaia, percebemos que cada folha pequena - parte da maior - tem o mesmo formato que a folha inteira. Dizemos que a folha da samambaia é auto-semelhante. $\mathrm{O}$ mesmo acontece com os fractais: podemos aumentá-los muitas vezes e depois de cada passo veremos a mesma forma, que é característica daquele fractal em particular.

Apesar de ser uma grande área de matemática, a Geometria Fractal é pouco estudada, ou não é estudada, durante o curso de graduação em matemática, principalmente nos cursos de licenciatura em Matemática.

Um dos fractais mais conhecidos é a famosa Curva de Koch, ou Floco de Neve ele tem início em um triângulo equilátero e a cada estágio temos que os segmentos são divididos em 3 partes, é construído um triângulo equilátero com base o segmento central dessa divisão e depois é retirado esse segmento central. Como na figura 1.

\section{Figura 1}

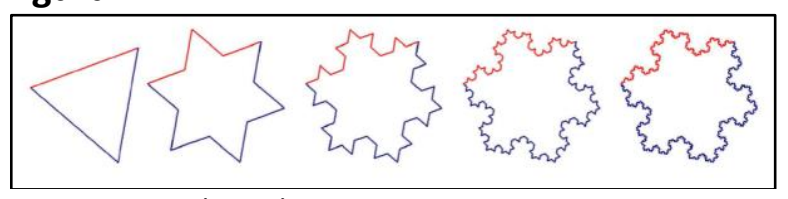

Fonte: Nunes (2016)

Como na Curva de Koch, um fractal pode ter origem em uma figura geométrica simples, como triângulos, quadrados, etc. e a partir disto, podemos aplicar infinitamente uma característica de repetição.

Em Jacomeli, Souza e Uribe (2019), o fractal construído se baseia na aplicação do teorema de Napoleão.

Teorema de Napoleão: Seja $\triangle A B C$ um triângulo arbitrário, se em seus lados forem construídos triângulos equiláteros, os ortocentros desses triângulos formará um novo triângulo equilátero.

A construção se inicia aplicando o teorema de Napoleão em um triângulo equilátero, obtendo a seguinte figura:

\section{Figura 2}

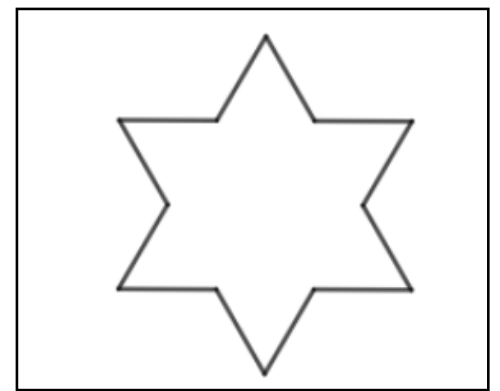

Fonte: Jacomeli, Souza e Uribe (2019)

A figura obtida é semelhante ao passo 1 do floco de neve. Cada ponta da figura 2 é um triângulo equilátero e pode ser aplicado novamente o teorema de Napoleão, obtendo assim a figura 3 .

Figura 3

Fonte: Jacomeli, Souza e Uribe (2019)

A ideia de Jacomeli, Souza e Uribe (2019), foi aplicar o teorema de Napoleão sucessivamente obtendo assim um fractal cuja área é finita e perímetro desse fractal na aplicação infinita do Teorema de Napoleão, chegando que o perímetro do fractal é divergente, ou seja, a soma dos perímetros 
tende ao infinito e a área é convergente chegando que

$$
A=\frac{7 \sqrt{3}}{18} l^{2}
$$

sendo $I$ o comprimento dos lados do triângulo inicial.

Ainda, um fractal pode ser representado também em um plano tridimensional, como é o caso do Tetraedro de Sierpinski (figura 4).

\section{Figura 4}

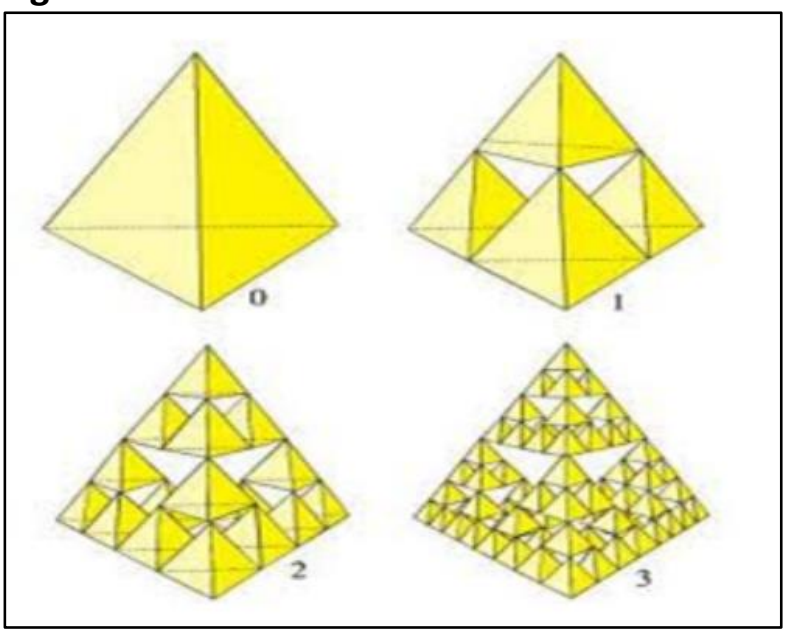

Fonte: Moura (2016)

Este fractal no plano tridimensional, tem início em um tetraedro regular, a partir dos pontos médios de suas arestas é formados 4 novos tetraedros menores (sendo os vértices desses tetraedros os pontos médios das arestas e o vértice do tetraedro inicial, como visto na figura 4). Após isso é retirado toda a parte central não pertencente a esses tetraedros menores. Repetindo esse processo novamente nos demais tetraedros que vão se formando.

Dessa forma, o objetivo desse trabalho é construir um fractal em um plano tridimensional, a partir de um tetraedro regular, com uma característica de repetição semelhante ao Teorema de Napoleão. Logo após isso, iremos estudar as características, como a área das superfícies e volume da figura.

\section{METODOLOGIA}

O presente trabalho faz parte da atividade de pesquisa individual do grupo PET Conexões de Saberes Matemática UFMS/CPTL, que possui como objetivo principal relembrar e/ou aprofundar o conhecimento em conceitos matemáticos que são poucos estudados, ou não estudados durante o curso de graduação.

Por fim, foi estudada uma forma para construir um fractal semelhante em um plano tridimensional. Logo, além de iniciar com um triângulo equilátero, iremos utilizar um tetraedro regular, e assim, iremos construir novos tetraedros regulares em suas faces, utilizando os pontos médios de suas arestas.

\section{RESULTADOS E DISCUSSÕES}

Um tetraedro regular possui 4 faces de formato triangular, sendo que esses triângulos são equiláteros e congruentes entre si. Vamos considerar um tetraedro de aresta $l$ e chamaremos de estágio 1 da construção do fractal.

Figura 5

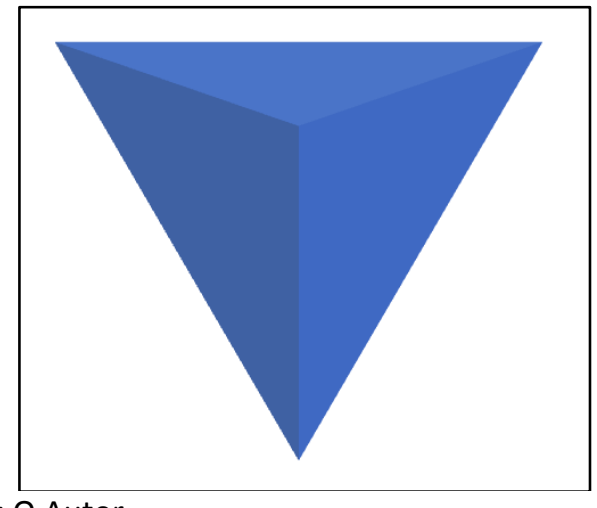

Fonte: O Autor.

No estágio 1 temos que a área e o volume são dados por:

$$
\begin{aligned}
& A_{1}=l^{2} \frac{\sqrt{3}}{2} \\
& V_{1}=\frac{\sqrt{2}}{12} l^{3} .
\end{aligned}
$$

Pelo fato das faces serem triângulos equiláteros, então tomamos os pontos 
médios das arestas desses triângulos, esses pontos formam um triângulo equilátero com lado de comprimento igual a metade do comprimento dos lados do triângulo anterior conforme a figura 6 .

\section{Figura 6}

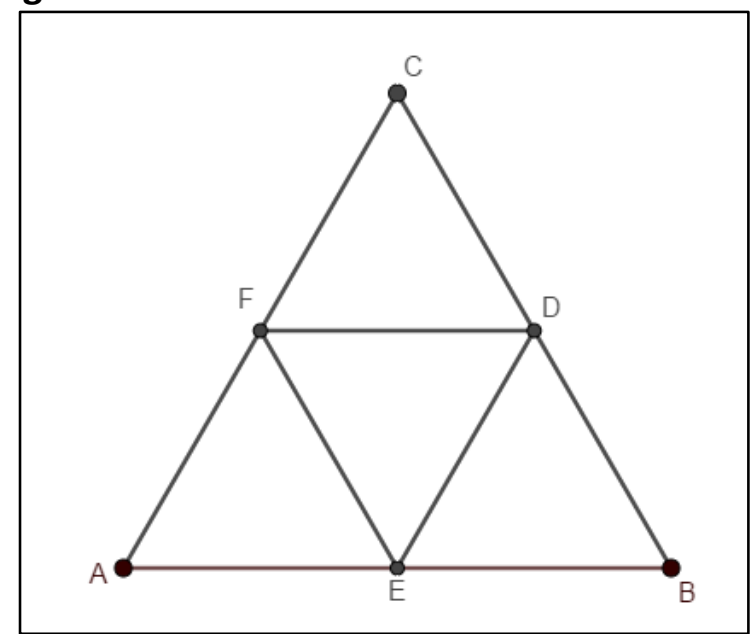

Fonte: O Autor.

Dem. Seja $A B C$ um triângulo equilátero, e seja $\mathrm{D}, \mathrm{E}$ e $\mathrm{F}$ pontos médios das arestas do triângulo, como na figura 6 .

Logo, temos

$$
\left\{\begin{array}{l}
\overline{A F}=\frac{1}{2} \overline{A C} \\
\overline{A E}=\frac{1}{2} \overline{A B} \\
\overline{F A E}=\overline{C A B}
\end{array}\right.
$$

Então, temos que os triângulos $A B C$ e AFE são semelhantes, logo

$$
\overline{F E}=\frac{1}{2} \overline{B C}
$$

Analogamente, temos que

$$
\left\{\begin{array}{l}
\overline{F D}=\frac{1}{2} \overline{A B} \\
\overline{D E}=\frac{1}{2} \overline{A C}
\end{array}\right.
$$

E, como $\overline{A B}=\overline{A C}=\overline{B C}$, então temos

que

$$
\overline{F E}=\overline{F D}=\overline{D E},
$$

Portanto o triângulo DEF é equilátero.

Dessa forma, com esses triângulos equiláteros menores nas faces do tetraedro podemos construir novos tetraedros regulares com suas bases nesses triângulos como mostra na figura 7 .
Figura 7

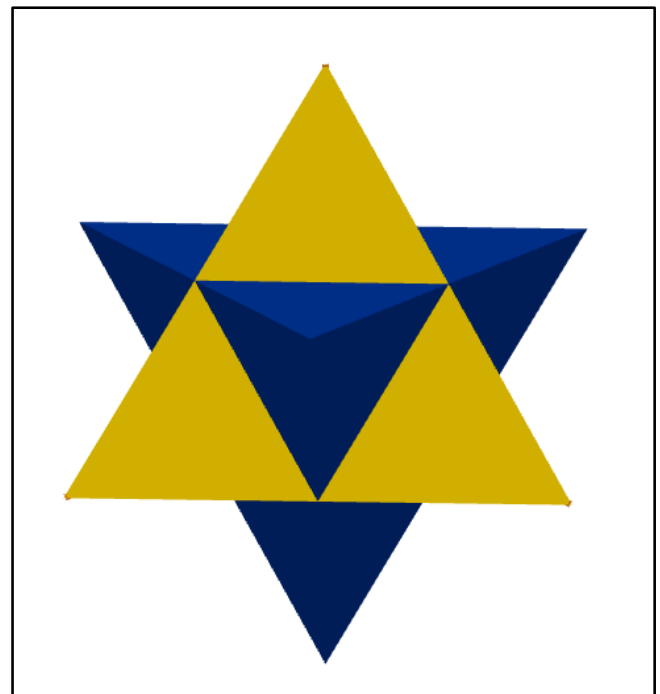

Fonte: O Autor.

$\mathrm{Na}$ figura 7 temos que o tetraedro azul é o tetraedro regular inicial, e os tetraedros amarelos são os tetraedros criados a partir dos triângulos equiláteros formados pelos pontos médios das arestas das faces. A figura 7 é o estágio 2 da construção do fractal.

No estágio 2 temos que o volume é dado pela soma do volume do estágio com os volumes dos novos tetraedros formados, ou seja:

$$
V_{2}=V_{1}+4 \frac{\sqrt{2}}{12}\left(\frac{l}{2}\right)^{3}=V_{1}+\frac{\sqrt{2}}{24} l^{3} .
$$

Consequentemente, em toda a figura, teremos novas faces triangulares, logo podemos repetir o mesmo processo e assim surgir novos tetraedros, agora com face com a proporção de $\frac{1}{4}$ em relação as faces do tetraedro inicial, temos assim o estágio 3 do fractal e é mostrado na figura 8. 
Figura 8

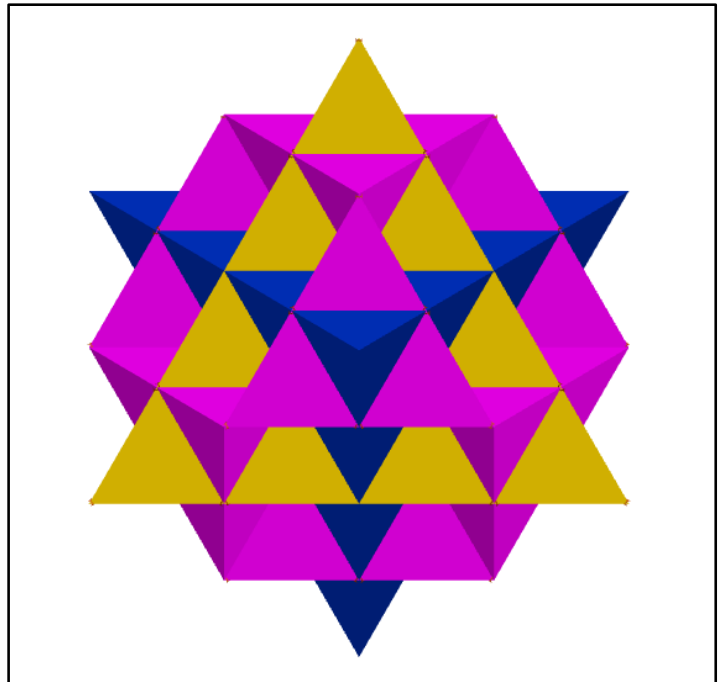

Fonte: O Autor.

No estágio 3 , temos que o volume da figura é a soma do volume do estágio $2 \mathrm{com}$ o volume dos novos tetraedros formados, ou seja:

$$
V_{3}=V_{2}+24 \frac{\sqrt{2}}{12}\left(\frac{l}{4}\right)^{3}=V_{2}+\frac{\sqrt{2}}{12} l^{3} \frac{2^{3} 3^{1}}{2^{6}}
$$

Agora, na figura formada no estágio 3, teremos novas faces triangulares, com proporção de $\frac{1}{8}$ em relação ao tetraedro inicial, logo poderemos repetir esse processo novamente, e assim sucessivamente.

Dessa maneira, teremos uma figura final que ao ir aproximando, independente de qual parte da figura, iremos encontrar sempre uma face triangular gerando um novo tetraedro regular, como na figura 9.

\section{Figura 9}

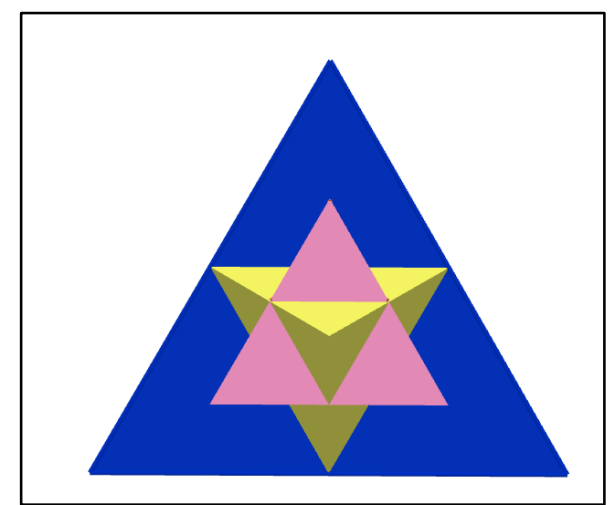

Fonte: O Autor.

Então, obtemos um fractal em um plano tridimensional na qual possui a característica geral de que cada face triângulo externa da figura é colocado um novo tetraedro na medida em que sua base é composta pelo triângulo formado pelos pontos médios da face.

\section{1. Áreas das Faces}

Seja uma face do tetraedro inicial, ao criarmos o triângulo formado pelos pontos médios da aresta, teremos a divisão da face em 4 triângulos congruentes, depois disso, é criado um tetraedro sob o triângulo central, um tetraedro possui 4 faces, porem a base do tetraedro será uma parte interna a figura geral, dessa forma temos que a cada face triangular, no estágio seguinte, teremos a formação de 6 novas faces triangulares, sendo 3 na face inicial e 3 no novo tetraedro formado, e assim sucessivamente para cada estágio do fractal.

\section{Figura 10}

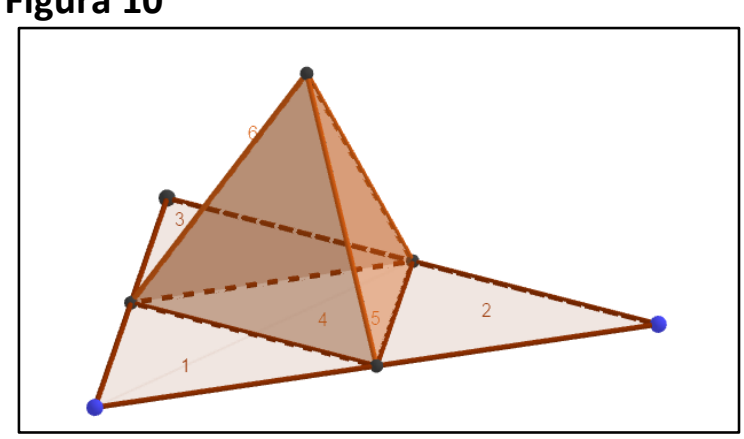

Fonte: O Autor.

Logo, seja I o comprimento do lado do tetraedro inicial, então temos quadro 1.

Quadro 1.

\begin{tabular}{|c|c|c|}
\hline Estágio & $\mathrm{N}^{\circ}$ de Faces & $\begin{array}{c}\text { Proporção } \\
\text { das faces }\end{array}$ \\
\hline 1 & $4.6^{0}$ & $\mathrm{l}$ \\
\hline 2 & $4.6^{1}=24$ & $\frac{l}{2}$ \\
\hline 3 & $\begin{array}{l}24.6=4.6^{2} \\
=144\end{array}$ & $\frac{l}{2^{2}}$ \\
\hline$\ldots$ & $\ldots$ & $\ldots$ \\
\hline $\mathrm{n}$ & $4.6^{n-1}$ & $\frac{l}{2^{n-1}}$ \\
\hline
\end{tabular}

Fonte: O Autor.

Agora, temos que a área de um triângulo equilátero é dada por 


$$
A=l^{2} \frac{\sqrt{3}}{4}
$$

Dem. Seja $A B C$ um triângulo equilátero de lado $I$, como na figura 11 .

\section{Figura 11}

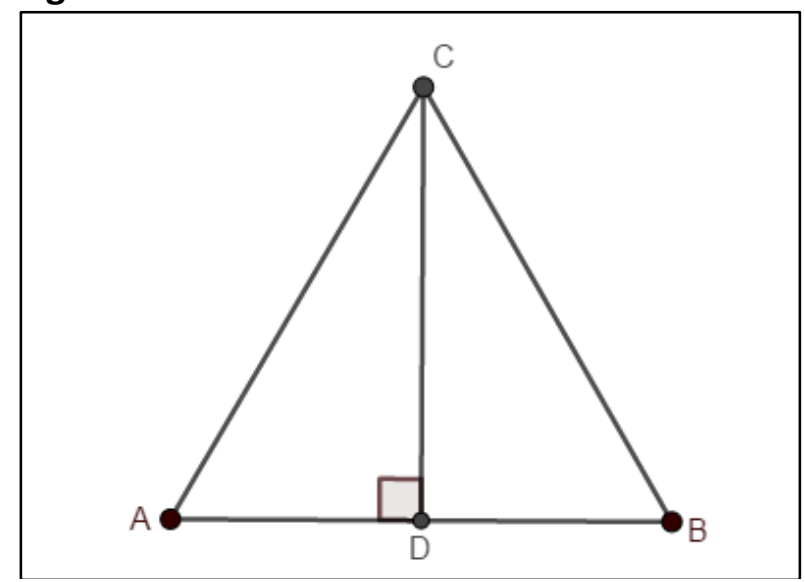

Fonte: O Autor.

Seja, $\overline{C D}$ a altura do triângulo, logo pelo Teorema de Pitágoras, temos:

Ou seja,

$$
(\overline{A C})^{2}=(\overline{C D})^{2}+(\overline{A D})^{2}
$$

$$
(\overline{C D})^{2}=(\overline{A C})^{2}-(\overline{A D})^{2}
$$

Sabendo que $\overline{A C}=l$ e que como o triângulo $A B C$ é equilátero. Com o ponto $D$ ponto médio de $\overline{A B}$ logo $\overline{A D}=\frac{l}{2}$. Assim,

$$
\overline{C D}=\frac{l \sqrt{3}}{2}
$$

Então, a área do triângulo será de

$$
A=\frac{\overline{A B} \cdot \overline{C D}}{2}=l^{2} \frac{\sqrt{3}}{4}
$$

Então temos que a soma das áreas das faces de cada estágio é dada por

$$
\begin{gathered}
A_{1}=4 l^{2} \frac{\sqrt{3}}{4} \\
A_{2}=24\left(\frac{l}{2}\right)^{2} \frac{\sqrt{3}}{4} \\
A_{3}=144\left(\frac{l}{4}\right)^{2} \frac{\sqrt{3}}{4}
\end{gathered}
$$

Logo, a soma das áreas das faces no nésimo estágio será dada por:

$$
A_{n}=4 \cdot 6^{n-1}\left(\frac{l}{2^{n-1}}\right)^{2} \frac{\sqrt{3}}{4}=
$$

$$
2^{n-1} 3^{n-1} \frac{l^{2}}{2^{2 n-2}} \sqrt{3}=
$$

$$
\frac{2^{n-1} 3^{n-1} l^{2} 4}{2^{2 n}} \sqrt{3}=
$$

$$
\frac{3^{n-1} 4 l^{2} \sqrt{3}}{2^{n+1}}=\frac{2 l^{2} \sqrt{3}}{3}\left(\frac{3}{2}\right)^{n}
$$

Temos então uma sequência numérica onde o termo geral é dado por

$$
A_{n}=\frac{2 l^{2} \sqrt{3}}{3}\left(\frac{3}{2}\right)^{n}
$$

Ou seja, é uma progressão geométrica de razão $3 / 2$, logo temos que

$$
\lim _{n \rightarrow \infty} A_{n}=\lim _{n \rightarrow \infty} \frac{2 l^{2} \sqrt{3}}{3}\left(\frac{3}{2}\right)^{n}=+\infty
$$

Portanto, quanto maior o estágio do fractal maior será a soma da área das faces da figura.

\subsection{Volume da Figura}

Voltando agora no tetraedro inicial, logo teremos que o volume total será de $V_{1}$, no estágio 2 teremos ainda $01^{\circ}$ tetraedro mas teremos adicionados mais 4 tetraedros menores, logo o volume será a soma do volume do tetraedro inicial $\left(V_{1}\right)$ mais a soma dos volumes dos 4 tetraedros menores. Assim, temos que a cada estágio o volume sempre irá aumentar os volumes dos novos tetraedros.

Agora, sabendo que em cada face irá acrescentar 1 novo tetraedro, temos o seguinte quadro:

Quadro 2

\begin{tabular}{|c|c|c|}
\hline Estágio & $\begin{array}{c}\text { Novos } \\
\text { Tetraedros }\end{array}$ & $\begin{array}{c}\text { Proporção } \\
\text { dos lados }\end{array}$ \\
\hline 1 & 1 & $l$ \\
\hline 2 & 4 & $\frac{l}{2}$ \\
\hline 3 & 24 & $\frac{l}{4}$ \\
\hline 4 & 144 & $\frac{l}{8}$ \\
\hline
\end{tabular}

Fonte: O Autor.

A fórmula do volume de um tetraedro é dada por: 


$$
V=\frac{\sqrt{2}}{12} l^{3}
$$

Demonstração: um tetraedro regular é uma pirâmide com as 4 faces triangulares congruentes.

Dessa forma, temos que o volume do tetraedro é dado por:

$$
V=\frac{1}{3} \cdot A \cdot h
$$

Sendo A a área da base do tetraedro e $\mathrm{h}$ a altura.

Sabendo que a base do tetraedro é um triângulo equilátero de lado $I$. Então observemos que:

$$
A=l^{2} \frac{\sqrt{3}}{4}
$$

E ainda, seja $h$ a altura do tetraedro, assim:

$$
h=\frac{l \sqrt{6}}{3}
$$

Consequentemente,

$$
V=\frac{1}{3} \cdot A \cdot h=\frac{1}{3} l^{2} \frac{\sqrt{3}}{4} \frac{l \sqrt{6}}{3}=\frac{\sqrt{2}}{12} l^{3}
$$

Então, seja $V_{i}$ o volume do estágio $i$.

$$
\begin{gathered}
V_{1}=\frac{\sqrt{2}}{12} l^{3} \\
V_{2}=V_{1}+4 \frac{\sqrt{2}}{12}\left(\frac{l}{2}\right)^{3}=V_{1}+\frac{\sqrt{2}}{12} l^{3} \frac{2^{2} 3^{0}}{2^{3}} \\
V_{3}=V_{2}+24 \frac{\sqrt{2}}{12}\left(\frac{l}{4}\right)^{3}=V_{2}+\frac{\sqrt{2}}{12} l^{3} \frac{2^{3} 3^{1}}{2^{6}} \\
V_{4}=V_{3}+144 \frac{\sqrt{2}}{12}\left(\frac{l}{8}\right)^{3}=3+\frac{\sqrt{2}}{12} l^{3} \frac{2^{4} 3^{2}}{2^{9}}
\end{gathered}
$$

Assim teremos,

$$
\begin{array}{r}
V_{n}=V_{n-1}+\frac{\sqrt{2}}{12} l^{3}\left(\frac{2^{n} 3^{n-2}}{2^{3(n-1)}}\right) \\
=V_{n-1}+\frac{\sqrt{2}}{12} l^{3}\left(\frac{3}{4}\right)^{n} \frac{8}{9} \\
=V_{n-1}+\frac{2 \sqrt{2}}{27} l^{3}\left(\frac{3}{4}\right)^{n}
\end{array}
$$

Ou seja, temos que o volume da figura é representado por uma recorrência de $1^{\circ}$ ordem. Logo,

$$
V_{2}=V_{1}+\frac{2 \sqrt{2}}{27} l^{3}\left(\frac{3}{4}\right)^{2}
$$

$$
\begin{gathered}
V_{3}=V_{2}+\frac{2 \sqrt{2}}{27} l^{3}\left(\frac{3}{4}\right)^{3} \\
V_{4}=V_{3}+\frac{\sqrt{2}}{27} l^{3}\left(\frac{3}{4}\right)^{4} \\
\cdots \\
V_{n-1}=V_{n-2}+\frac{2 \sqrt{2}}{27} l^{3}\left(\frac{3}{4}\right)^{n-1} \\
V_{n}=V_{n-1}+\frac{2 \sqrt{2}}{27} l^{3}\left(\frac{3}{4}\right)^{n} \\
\text { Somando as igualdades temos, } \\
V_{n}=V_{1}+\frac{2 \sqrt{2}}{27} l^{3}\left(\left(\frac{3}{4}\right)^{1}+\left(\frac{3}{4}\right)^{2}+\left(\frac{3}{4}\right)^{3}+\cdots\right. \\
\left.+\left(\frac{3}{4}\right)^{n-1}+\left(\frac{3}{4}\right)^{n}\right)
\end{gathered}
$$

Temos então a soma de uma progressão geométrica com razão de $1 / 3$, assim

$$
\begin{aligned}
V_{n}=V_{1}+\frac{2 \sqrt{2}}{27} & l^{3} \frac{3}{4}\left(\frac{1-\left(\frac{3}{4}\right)^{n-2}}{1-\frac{3}{4}}\right) \\
& =V_{1}+\frac{2 \sqrt{2}}{27} l^{3} 3\left(1-\left(\frac{3}{4}\right)^{n-2}\right)
\end{aligned}
$$

Encontramos então o volume da figura no nésimo estágio. Dessa forma, o volume do fractal repetindo o processo infinitas vezes é dado por

$$
\begin{gathered}
\lim _{n \rightarrow \infty} V_{n}=\lim _{n \rightarrow \infty} V_{1}+\frac{2 \sqrt{2}}{27} l^{3} 3\left(1-\left(\frac{3}{4}\right)^{n-2}\right) \\
=V_{1}+\frac{2 \sqrt{2}}{27} l^{3} 3\left[\lim _{n \rightarrow \infty}\left(1-\left(\frac{3}{4}\right)^{n-2}\right)\right] \\
=V_{1}+3 \frac{2 \sqrt{2}}{27} l^{3} \\
=\frac{\sqrt{2}}{12} l^{3}+\frac{2 \sqrt{2}}{9} l^{3}=\frac{33 \sqrt{2}}{108} l^{3}
\end{gathered}
$$

Portanto, temos que o volume da figura, na aplicação infinita, será convergente, ou seja, o volume será igual a $\frac{33 \sqrt{2}}{108} l^{3}$.

\section{CONCLUSÃO}

Como visto em Jacomeli, Souza e Uribe (2019), podemos construir um fractal, em um plano bidimensional com perímetro divergente e área convergente, a partir de um triângulo equilátero e utilizando o Teorema de Napoleão. 
Neste trabalho vimos que podemos construir um fractal em um plano tridimensional, com início em um tetraedro regular e com uma característica de repetição semelhando ao Teorema de Napoleão. Após criado esse fractal pudemos analisar características como área da superfície e volume total desse fractal. Concluindo então que o a área será infinita e o volume será limitado.

Portanto, este trabalho é a parte final da pesquisa que englobou estudo e aprofundamento em conceitos como geometria, álgebra, cálculo e também um estudo de fractais em planos bidimensional e tridimensional.

\section{REFERÊNCIAS}

ALVES, D. S. Os teoremas esquecidos pelos professores de geometria plana do ensino médio. 2015. Dissertação (Mestrado Profissional) - Universidade Federal de Mato Grosso do Sul, Campo Grande, MS, 2015.

BRITO, A. J.; CARVALHO, D. L. História da matemática em atividades didáticas: Utilizando a história no Ensino de Geometria. Natal: EDUFRN, 2005.

GONZAGA, G. C. S. Teorema de Napoleão: origem, demonstração e aplicações. 2015. Dissertação (Mestrado em Matemática) Universidade Federal de Goiás, Goiânia, GO, 2015.

JURGENS, H.; PEITGEN, H.; SAUPE, D. The Language of Fractals. Scientific American, Aug., 1990. https://doi.org/10.1038/scientificamerican08 $\underline{90-60}$

JACOMELI, J. A. C.; SOUZA , F. P.; URIBE, E. B. O. FRACTAIS: Uma Aplicação Do Teorema De Napoleão. Colloquium Exactarum, v. 11, n. 3, p. 84-91, out. 2019.

https://doi.org/10.5747/ce.2019.v11.n3.e287

MACEDO, D. M. R. Resgatando alguns teoremas clássicos da geometria plana.
Dissertação (Mestrado) - Universidade Federal do Ceará, Juazeiro do Norte, CE, 2014.

MOURA, D. V., Introdução à Geometria Fractal. Dissertação (Mestrado Profissional em Matemática - PROFMAT) - Universidade Federal do Piauí, Piai,2016.

NUNES, R. S. R. Geometria fractal e aplicações. 2006. Dissertação (Mestrado em Ensino da Matemática) - Universidade do Porto, Departamento de Matemática Pura., PO, 2006.

SHENKER, O. R. Fractal geometry is not the geometry of nature. Studies in History and Philosophy of Science Part A., v. 25,n.6, p. 967-981,1994. https://doi.org/10.1016/00393681(94)90072-8 